Research Article

\title{
Perturbation to Noether Symmetries and Adiabatic Invariants for Birkhoffian Systems
}

\author{
Yi Zhang \\ College of Civil Engineering, Suzhou University of Science and Technology, Suzhou, Jiangsu 215011, China \\ Correspondence should be addressed to Yi Zhang; weidiezh@gmail.com
}

Received 4 January 2015; Revised 26 April 2015; Accepted 30 April 2015

Academic Editor: Jun-Juh Yan

Copyright ( 2015 Yi Zhang. This is an open access article distributed under the Creative Commons Attribution License, which permits unrestricted use, distribution, and reproduction in any medium, provided the original work is properly cited.

\begin{abstract}
Based on El-Nabulsi dynamical model for a non-conservative system, the problem of perturbation to Noether symmetries and adiabatic invariants of a Birkhoffian system under the action of a small disturbance is proposed and studied. Firstly, the El-NabulsiPfaff variational problem from extended exponentially fractional integral is presented and the El-Nabulsi-Birkhoff equations are established. Secondly, the definitions and the criterions criteria of the Noether symmetric transformations and quasisymmetric transformations of the Birkhoffian system are given, and the Noether theorems of the system are established, which reveal the inner relationship between the Noether symmetries and the conserved quantities. Thirdly, the perturbation of Noether symmetries under a small disturbance is studied, and corresponding adiabatic invariants are obtained. As special cases, the deductions in nonconservative Hamiltonian system and nonconservative Lagrangian system and standard Birkhoffian system are given. At the end of the paper, the case known as Hojman-Urrutia problem is discussed to investigate the Noether symmetries and the adiabatic invariants, the perturbation to Noether symmetries and the adiabatic invariants under El-Nabulsi dynamical model.
\end{abstract}

\section{Introduction}

In 1927, Birkhoff [1] proposed a kind of more general dynamical equations than Hamilton canonical equations and a kind of more general integral variational principle than Hamilton principle. The above results were extended to the case including the time by Santilli [2] who made the word Birkhoffian mechanics and pointed out that the Birkhoffian mechanics is the most general possible mechanics that can be constructed from the Hamiltonian mechanics via the transformation theory. In fact, all holonomic systems and nonholonomic systems can be incorporated into the Birkhoffian systems [3]. Galiullan pointed out $[4,5]$ that it is an important developmental direction of modern analytical mechanics to study the Birkhoffian mechanics. Mei pointed out that the Birkhoffian mechanics is a new development after quantum mechanics appears [6]. The study on the dynamics of Birkhoffian system has made a series of important results $[7,8]$.

The fractional variational problems combine the calculus of variations with the fractional calculus through inserting the fractional derivatives in the variational integrals. The fractional calculus of variations was introduced by Riewe in $[9$, 10] where he developed the nonconservative dynamics using fractional calculus. Since then, the fractional variational problems have been becoming a subject of current strong researches of applied mathematics, physics, dynamics, and control and have highly aroused concern by many scholars, for example, Klimek [11], Agrawal [12, 13], Atanacković [14, 15], Baleanu [16, 17], Torres [18, 19], Cresson [20], and their collaborators. In 2005, El-Nabulsi put forward a nonconservative dynamics model $[21,22]$ which is based on the definition of Riemann-Liouville fractional integral within the framework of fractional calculus and further presented the nonconservative dynamics models based on exponentially extended fractional integral [23] and periodically extended fractional integral [24], respectively. These models can be called El-Nabulsi dynamics models. The novelty of El-Nabulsi models is that the resulting Euler-Lagrange equations are simple and similar to the classic ones for nonconservative dynamical systems, with no fractional derivatives appearing, but with the presence of the fractional generalized external 
force acting on the system. Frederico and Torres [25, 26] studied the constants of motion for nonconservative system under the El-Nabulsi dynamics model. Recently, the ElNabulsi dynamics models were extended to the Birkhoffian systems by Zhang and his coworkers, and the Noether theorems of the Birkhoffian systems were established [27, 28].

For a dynamical system, there exists intimate relation between the integrability of the system and the variations in its symmetry and invariant under the action of small disturbance $[29,30]$, and therefore the researches on the perturbation to symmetries and adiabatic invariants are of great significance. A classical adiabatic invariant is a certain physical quantity that changes more slowly than some parameter of the system when the parameter varies very slowly. In fact, the parameter varying very slowly is equivalent to the action of small disturbance. Some important results for the study of perturbation to symmetries and adiabatic invariants for constrained mechanical systems have been obtained [3135]. Very recently, we studied the perturbation to Noether symmetries and adiabatic invariants for Birkhoffian systems based on El-Nabulsi dynamical models from the definition of Riemann-Liouville fractional integral [36]. In this paper, we will study the Noether symmetries and perturbation for ElNabulsi-Pfaff variational problem from extended exponentially fractional integral. The motivation for extending the results in [36] to its exponential counterpart is that the latter is intimately associated with quantum Bateman-FeshbachTikochinsky and Caldirola-Kanai damped harmonic oscillators which are explored extensively in literature $[23,37,38]$ and references therein.

The paper is organized in the following way. In Section 2, the El-Nabulsi-Pfaff-Birkhoff variational problem from extended exponentially fractional integral is presented. In Section 3, two basic formulae for variation of El-NabulsiPfaff action are given. In Section 4, the definitions and the criteria of the Noether symmetries are established, and the Noether symmetries and the conserved quantities of the system are studied in terms of the invariance of El-NabulsiPfaff action under the infinitesimal transformations. In Section 5, the perturbation of Noether symmetries under small disturbance is studied, and corresponding adiabatic invariants are given. As special cases, the exact invariants and the adiabatic invariants for nonconservative Hamiltonian systems and nonconservative Lagrangian systems under ElNabulsi models and classic Birkhoffian systems are deduced. In Section 6, the well-known Hojman-Urrutia problem [2] is taken as an example to illustrate the application of the results. We finish with Section 7 of conclusions.

\section{El-Nabulsi-Pfaff Variational Problem}

To study dynamics modeling of a nonconservative system, El-Nabulsi proposed the variational problem from extended exponentially fractional integral [23]. Now we extend the ElNabulsi model to a Birkhoffian system. And for a Birkhoffian system, a Pfaff variational problem from extended exponentially fractional integral can be defined as follows.
Find an extreme value problem of the integral functional

$$
\begin{aligned}
& S(\gamma)=\frac{1}{\Gamma(\alpha)} \int_{t_{1}}^{t_{2}}\left[R_{\mu}\left(\tau, a^{\nu}\right) \dot{a}^{\mu}-B\left(\tau, a^{\nu}\right)\right] \\
& \cdot(\cosh t-\cosh \tau)^{\alpha-1} d \tau
\end{aligned}
$$

with given boundary conditions

$$
\begin{array}{r}
\left.a^{\mu}\right|_{\tau=t_{1}}=a_{1}^{\mu}, \\
\left.a^{\mu}\right|_{\tau=t_{2}}=a_{2}^{\mu}, \\
(\mu=1,2, \ldots, 2 n),
\end{array}
$$

where $\gamma$ is a curve, $B=B\left(\tau, a^{\nu}\right)$ is the Birkhoffian, $R_{\mu}=$ $R_{\mu}\left(\tau, a^{\nu}\right)$ are Birkhoff's functions, $a^{\mu}$ are Birkhoff's variables, $\dot{a}^{\mu}=d a^{\mu} / d \tau, \Gamma$ is the Euler Gamma function, $0<\alpha \leq 1$, $\tau$ is the intrinsic time, $t$ is the observer time, $\tau \neq t$, and the Birkhoffian $B$ and Birkhoff's functions $R_{\mu}$ are $C^{2}$ functions with respect to all their arguments.

The above problem can be called the El-Nabulsi-Pfaff variational problem from extended exponentially fractional integral. Functional (1) can be called the El-Nabulsi-Pfaff action. If $\alpha=1$, then this problem is reduced to the classical Pfaff variational problem.

If functional (1) reaches its extreme value at $a^{\mu}=a^{\mu}(\tau)$, then we have [28]

$$
\begin{array}{r}
\left(\frac{\partial R_{v}}{\partial a^{\mu}}-\frac{\partial R_{\mu}}{\partial a^{\nu}}\right) \dot{a}^{\nu}-\frac{\partial B}{\partial a^{\mu}}-\frac{\partial R_{\mu}}{\partial \tau}=\frac{(1-\alpha) \sinh \tau}{\cosh t-\cosh \tau} R_{\mu} \\
(\mu=1,2, \ldots, 2 n) .
\end{array}
$$

Equations (3) can be called the El-Nabulsi-Birkhoff equations for the El-Nabulsi-Pfaff variational problem from extended exponentially fractional integral.

Equations (3) are universal. Firstly, if we take $\alpha=1$, then

(3) can be reduced to the standard Birkhoff equations $[2,3]$ :

$$
\begin{aligned}
&\left(\frac{\partial R_{v}}{\partial a^{\mu}}-\frac{\partial R_{\mu}}{\partial a^{\nu}}\right) \dot{a}^{\nu}-\frac{\partial B}{\partial a^{\mu}}-\frac{\partial R_{\mu}}{\partial \tau}=0, \\
&(\mu=1,2, \ldots, 2 n) .
\end{aligned}
$$

Secondly, if we take

$$
\begin{aligned}
a^{\mu} & = \begin{cases}q^{\mu} & (\mu=1,2, \ldots, n) \\
p_{\mu-n} & (\mu=n+1, \ldots, 2 n),\end{cases} \\
R_{\mu} & = \begin{cases}p_{\mu} & (\mu=1,2, \ldots, n) \\
0 & (\mu=n+1, \ldots, 2 n),\end{cases} \\
B & =H,
\end{aligned}
$$


where $H=H(\tau, \mathbf{p}, \mathbf{q})$ is the Hamiltonian, $q^{k}$ are the generalized coordinates, and $p_{k}$ are the generalized momenta, then (3) give

$$
\begin{array}{r}
\dot{q}^{k}=\frac{\partial H}{\partial p_{k}}, \\
\dot{p}_{k}=-\frac{\partial H}{\partial q^{k}}-\frac{(1-\alpha) \sinh \tau}{\cosh t-\cosh \tau} p_{k}, \\
(k=1,2, \ldots, n) .
\end{array}
$$

Equations (6) are the El-Nabulsi-Hamilton canonical equations. And then, by means of the Legendre transformations

$$
H=p_{k} \dot{q}^{k}-L
$$

where $L=L(\tau, \mathbf{q}, \dot{\mathbf{q}})$ is the Lagrangian, (6) give

$$
\frac{\partial L}{\partial q^{k}}-\frac{d}{d \tau} \frac{\partial L}{\partial \dot{q}^{k}}=\frac{(1-\alpha) \sinh \tau}{\cosh t-\cosh \tau} \frac{\partial L}{\partial \dot{q}^{k}},
$$

$$
(k=1,2, \ldots, n) .
$$

Equations (8) can be called the El-Nabulsi-Euler-Lagrange equations.

\section{Basic Formulae for Variation of El-Nabulsi-Pfaff Action}

Introduce the infinitesimal transformations of $r$-parameter finite transformation group

$$
\begin{aligned}
\bar{\tau} & =\tau+\Delta \tau, \\
\bar{a}^{\mu}(\bar{\tau}) & =a^{\mu}(\tau)+\Delta a^{\mu}, \quad(\mu=1,2, \ldots, 2 n)
\end{aligned}
$$

or their expansion formula

$$
\begin{aligned}
\bar{\tau} & =\tau+\varepsilon_{\sigma} \xi_{0}^{\sigma}\left(\tau, a^{\nu}\right), \\
\bar{a}^{\mu}(\bar{\tau}) & =a^{\mu}(\tau)+\varepsilon_{\sigma} \xi_{\mu}^{\sigma}\left(\tau, a^{\nu}\right), \quad(\mu=1,2, \ldots, 2 n),
\end{aligned}
$$

where $\varepsilon_{\sigma}$ are infinitesimal parameters and $\xi_{0}^{\sigma}$, $\xi_{\mu}^{\sigma}$ are the infinitesimal generators or the generating functions of the infinitesimal transformations. Under the action of the infinitesimal transformations (9), the curve $\gamma$ will be transformed into a neighbor curve $\bar{\gamma}$, the El-Nabulsi-Pfaff action (1) is transformed into $S(\bar{\gamma})$, and, assuming that $\Delta S$ is the main linear part relative to $\varepsilon$ in the difference $S(\bar{\gamma})-S(\gamma)$, then we have [28]

$$
\begin{aligned}
\Delta S & =\frac{1}{\Gamma(\alpha)} \int_{t_{1}}^{t_{2}}\left\{\left[\left(\frac{\partial R_{\mu}}{\partial \tau} \dot{a}^{\mu}-\frac{\partial B}{\partial \tau}\right) \Delta \tau\right.\right. \\
& +\left(\frac{\partial R_{\mu}}{\partial a^{\nu}} \dot{a}^{\mu}-\frac{\partial B}{\partial a^{\nu}}\right) \Delta a^{\nu}+R_{\mu} \Delta \dot{a}^{\mu} \\
& \left.+\left(R_{\mu} \dot{a}^{\mu}-B\right) \frac{d}{d \tau} \Delta \tau\right] \cdot(\cosh t-\cosh \tau)^{\alpha-1} \\
& -\left(R_{\mu} \dot{a}^{\mu}-B\right)(\alpha-1) \Delta \tau(\cosh t-\cosh \tau)^{\alpha-2} \\
& \cdot \sinh \tau\} d \tau .
\end{aligned}
$$

Notice that there is the relationship [8] between the nonisochronous variation $\Delta$ and the isochronous variation $\delta$ as follows:

$$
\begin{aligned}
\delta \mathrm{a}^{\mu} & =\Delta a^{\mu}-\dot{a}^{\mu} \Delta \tau, \\
\Delta \dot{a}^{\mu} & =\frac{d}{d \tau}\left(\Delta a^{\mu}\right)-\dot{a}^{\mu} \frac{d}{d \tau}(\Delta \tau) .
\end{aligned}
$$

From (12) and making use of formula (10), we can rewrite (11) as follows:

$$
\begin{aligned}
\Delta S & =\frac{1}{\Gamma(\alpha)} \\
& \cdot \int_{t_{1}}^{t_{2}} \varepsilon_{\sigma}\left\{\frac{d}{d \tau}\left[\left(R_{\mu} \xi_{\mu}^{\sigma}-B \xi_{0}^{\sigma}\right)(\cosh t-\cosh \tau)^{\alpha-1}\right]\right. \\
& +\left[\left(\frac{\partial R_{v}}{\partial a^{\mu}}-\frac{\partial R_{\mu}}{\partial a^{\nu}}\right) \dot{a}^{\nu}-\frac{\partial B}{\partial a^{\mu}}-\frac{\partial R_{\mu}}{\partial \tau}\right. \\
& \left.-\frac{(1-\alpha) \sinh \tau}{\cosh t-\cosh \tau} R_{\mu}\right](\cosh t-\cosh \tau)^{\alpha-1}\left(\xi_{\mu}^{\sigma}\right. \\
& \left.\left.-\dot{a}^{\mu} \xi_{0}^{\sigma}\right)\right\} d \tau .
\end{aligned}
$$

Formulae (11) and (13) are two basic formulae for variation of El-Nabulsi-Pfaff action (1)

\section{Noether Symmetries and Conserved Quantities}

The Noether symmetry of the El-Nabulsi-Pfaff variational problem is an invariance of the El-Nabulsi-Pfaff action (1) under the infinitesimal transformations. Now, we give the definitions and criterions of the Noether symmetries of the variational problem from extended exponentially fractional integral for a Birkhoffian system. 
Definition 1. If the El-Nabulsi-Pfaff action (1) is a quasiinvariant of the infinitesimal transformations (9) (i.e., for each of the infinitesimal transformations (9), the relation

$$
\Delta S=-\frac{1}{\Gamma(\alpha)} \int_{t_{1}}^{t_{2}} \frac{d}{d \tau}(\Delta G) d \tau
$$

always holds, where $\Delta G=\varepsilon_{\sigma} G^{\sigma}, G^{\sigma}=G^{\sigma}\left(\tau, a^{\nu}\right)$ are gauge functions), then the infinitesimal transformations are called the Noether quasisymmetric transformations of the variational problem from extended exponentially fractional integral for a Birkhoffian system.

According to Definition 1 and formula (11), we can easily get the following criterion.

Criterion 1. If the infinitesimal transformations (9) satisfy the following condition,

$$
\begin{gathered}
\left(\frac{\partial R_{\mu}}{\partial \tau} \dot{a}^{\mu}-\frac{\partial B}{\partial \tau}\right) \Delta \tau+\left(\frac{\partial R_{\mu}}{\partial a^{\nu}} \dot{a}^{\mu}-\frac{\partial B}{\partial a^{\nu}}\right) \Delta a^{\nu}+R_{\mu} \Delta \dot{a}^{\mu} \\
+\left(R_{\mu} \dot{a}^{\mu}-B\right)\left(\frac{d}{d \tau} \Delta \tau+\frac{(1-\alpha) \sinh \tau}{\cosh t-\cosh \tau} \Delta \tau\right) \\
=-\frac{d}{d \tau}(\Delta G)(\cosh t-\cosh \tau)^{1-\alpha}
\end{gathered}
$$

then the transformations are the Noether quasisymmetric transformations in the sense of Definition 1 for the Birkhoffian system under study.

Condition (15) can be also written as $r$ equations:

$$
\begin{aligned}
& \left(\frac{\partial R_{\mu}}{\partial \tau} \dot{a}^{\mu}-\frac{\partial B}{\partial \tau}\right) \xi_{0}^{\sigma}+\left(\frac{\partial R_{\mu}}{\partial a^{\nu}} \dot{a}^{\mu}-\frac{\partial B}{\partial a^{\nu}}\right) \xi_{v}^{\sigma} \\
& \quad+R_{\mu}\left(\dot{\xi}_{\mu}^{\sigma}-\dot{a}^{\mu} \dot{\xi}_{0}^{\sigma}\right) \\
& \quad+\left(R_{\mu} \dot{a}^{\mu}-B\right)\left(\dot{\xi}_{0}^{\sigma}+\frac{(1-\alpha) \sinh \tau}{\cosh t-\cosh \tau} \xi_{0}^{\sigma}\right) \\
& =-\dot{G}^{\sigma}(\cosh t-\cosh \tau)^{1-\alpha}, \quad(\sigma=1,2, \ldots, r) .
\end{aligned}
$$

When $r=1$, (16) can also be called the Noether identity of the variational problem from extended exponentially fractional integral for a Birkhoffian system.

Under the El-Nabulsi dynamics model, the Noether conserved quantity for a Birkhoffian system can be directly led by the Noether symmetry of the system; we have the following theorem.

Theorem 2. For the Birkhoffian system (3), if the infinitesimal transformations (9) are the Noether quasisymmetric transformations in the sense of Definition 1, then the system admits $r$ linearly independent conserved quantities, such as

$$
\begin{array}{r}
I^{\sigma}=\left(R_{\mu} \xi_{\mu}^{\sigma}-B \xi_{0}^{\sigma}\right)(\cosh t-\cosh \tau)^{\alpha-1}+G^{\sigma}=c^{\sigma}, \\
(\sigma=1,2, \ldots, r) .
\end{array}
$$

The conserved quantities (17) are exact invariants. The Noether theorem reveals the relationship between the Noether symmetries and the exact invariants for the undisturbed Birkhoffian system (3) under the El-Nabulsi dynamics model.

Theorem 2 is universal. Firstly, if $\alpha=1$, Theorem 2 is reduced to the Noether theorem for a classical Birkhoffian system.

Theorem 3. For the classical Birkhoffian system (4), if there is a gauge function $G^{\sigma}\left(\tau, a^{\nu}\right)$ that makes the generators $\xi_{0}^{\sigma}\left(\tau, a^{\nu}\right), \xi_{\mu}^{\sigma}\left(\tau, a^{\nu}\right)$ of infinitesimal transformations (9) satisfy the following conditions,

$$
\begin{aligned}
&\left(\frac{\partial R_{\mu}}{\partial \tau} \dot{a}^{\mu}-\frac{\partial B}{\partial \tau}\right) \xi_{0}^{\sigma}+\left(\frac{\partial R_{\mu}}{\partial a^{\nu}} \dot{a}^{\mu}-\frac{\partial B}{\partial a^{\nu}}\right) \xi_{\nu}^{\sigma} \\
&+R_{\mu}\left(\dot{\xi}_{\mu}^{\sigma}-\dot{a}^{\mu} \dot{\xi}_{0}^{\sigma}\right)+\left(R_{\mu} \dot{a}^{\mu}-B\right) \dot{\xi}_{0}^{\sigma}=-\dot{G}^{\sigma}, \\
&(\sigma=1,2, \ldots, r)
\end{aligned}
$$

then the system admits $r$ linearly independent conserved quantities, such as

$$
I^{\sigma}=R_{\mu} \xi_{\mu}^{\sigma}-B \xi_{0}^{\sigma}+G^{\sigma}=c^{\sigma}, \quad(\sigma=1,2, \ldots, r)
$$

Secondly, let us discuss a nonconservative Hamiltonian system under the El-Nabulsi dynamics model. Theorem 2 is reduced to the following theorem.

Theorem 4. For the nonconservative Hamiltonian system (6) under the El-Nabulsi dynamics model, if there is a gauge function $G^{\sigma}\left(\tau, q^{s}, p_{s}\right)$ that makes the generators $\xi_{0}^{\sigma}\left(\tau, q^{s}, p_{s}\right)$, $\xi_{k}^{\sigma}\left(\tau, q^{s}, p_{s}\right)$, and $\eta_{k}^{\sigma}\left(\tau, q^{s}, p_{s}\right)$ of infinitesimal transformations (9) satisfy the following conditions,

$$
\begin{aligned}
& -\frac{\partial H}{\partial \tau} \xi_{0}^{\sigma}-\frac{\partial H}{\partial q^{k}} \xi_{k}^{\sigma}+\left(\dot{q}^{k}-\frac{\partial H}{\partial p_{k}}\right) \eta_{k}^{\sigma}+p_{k}\left(\dot{\xi}_{k}^{\sigma}-\dot{q}^{k} \dot{\xi}_{0}^{\sigma}\right) \\
& +\left(p_{k} \dot{q}^{k}-H\right)\left(\dot{\xi}_{0}^{\sigma}+\frac{(1-\alpha) \sinh \tau}{\cosh t-\cosh \tau} \xi_{0}^{\sigma}\right) \\
& =-\dot{G}^{\sigma}(\cosh t-\cosh \tau)^{1-\alpha}, \quad(\sigma=1,2, \ldots, r)
\end{aligned}
$$

then the system admits $r$ linearly independent conserved quantities, such as

$$
\begin{array}{r}
I^{\sigma}=\left(p_{k} \xi_{k}^{\sigma}-H \xi_{0}^{\sigma}\right)(\cosh t-\cosh \tau)^{\alpha-1}+G^{\sigma}=c^{\sigma}, \\
(\sigma=1,2, \ldots, r),
\end{array}
$$

where $\Delta \tau=\varepsilon_{\sigma} \xi_{0}^{\sigma}, \Delta q^{k}=\varepsilon_{\sigma} \xi_{k}^{\sigma}$, and $\Delta p_{k}=\varepsilon_{\sigma} \eta_{k}^{\sigma}$.

Again, let us discuss a nonconservative Lagrange system under the El-Nabulsi dynamics model. Theorem 2 is reduced to the following theorem.

Theorem 5. For the nonconservative Lagrangian system (8) under the El-Nabulsi dynamics model, if there is a gauge function $G^{\sigma}\left(\tau, q^{s}, \dot{q}^{s}\right)$ that makes the generators $\xi_{0}^{\sigma}\left(\tau, q^{s}, \dot{q}^{s}\right)$ 
and $\xi_{k}^{\sigma}\left(\tau, q^{s}, \dot{q}^{s}\right)$ of infinitesimal transformations (9) satisfy the following conditions,

$$
\begin{aligned}
\frac{\partial L}{\partial \tau} \xi_{0}^{\sigma} & +\frac{\partial L}{\partial q^{k}} \xi_{k}^{\sigma}+\frac{\partial L}{\partial \dot{q}^{k}}\left(\dot{\xi}_{k}^{\sigma}-\dot{q}^{k} \dot{\xi}_{0}^{\sigma}\right) \\
& +L\left(\dot{\xi}_{0}^{\sigma}+\frac{(1-\alpha) \sinh \tau}{\cosh t-\cosh \tau} \xi_{0}^{\sigma}\right) \\
= & -\dot{G}^{\sigma}(\cosh t-\cosh \tau)^{1-\alpha}, \quad(\sigma=1,2, \ldots, r),
\end{aligned}
$$

then the system admits $r$ linearly independent conserved quantities, such as

$$
\begin{aligned}
I^{\sigma}= & {\left[L \xi_{0}^{\sigma}+\frac{\partial L}{\partial \dot{q}_{k}}\left(\xi_{k}^{\sigma}-\dot{q}_{k} \xi_{0}^{\sigma}\right)\right](\cosh t-\cosh \tau)^{\alpha-1} } \\
& +G^{\sigma}=c^{\sigma}, \quad(\sigma=1,2, \ldots, r) .
\end{aligned}
$$

\section{Perturbation to Noether Symmetries and Adiabatic Invariants}

For the Birkhoffian system (3) under the El-Nabulsi dynamics model, if $\xi_{0}^{\sigma 0}$ and $\xi_{\mu}^{\sigma 0}$ correspond to the Noether quasisymmetric transformations in the sense of Definition 1, then the system admits the following exact invariants:

$$
\begin{array}{r}
I_{0}^{\sigma}=\left(R_{\mu} \xi_{\mu}^{\sigma 0}-B \xi_{0}^{\sigma 0}\right)(\cosh t-\cosh \tau)^{\alpha-1}+G^{\sigma 0}=c^{\sigma}, \\
(\sigma=1,2, \ldots, r) .
\end{array}
$$

Suppose that the Birkhoffian system (3) is disturbed by small forces of perturbation $\rho Q_{\mu}$, then the differential equations of motion of the system will be changed into the following equations:

$$
\begin{aligned}
& \left(\frac{\partial R_{v}}{\partial a^{\mu}}-\frac{\partial R_{\mu}}{\partial a^{\nu}}\right) \dot{a}^{\nu}-\frac{\partial B}{\partial a^{\mu}}-\frac{\partial R_{\mu}}{\partial \tau} \\
& \quad=\frac{(1-\alpha) \sinh \tau}{\cosh t-\cosh \tau} R_{\mu}+\rho Q_{\mu}, \quad(\mu=1,2, \ldots, 2 n) .
\end{aligned}
$$

For undisturbed system (3), the conserved quantities (24) are exact invariants, but not for disturbed system (25). Assume that the disturbed generators $\xi_{0}^{\sigma}$ and $\xi_{\mu}^{\sigma}$ of the infinitesimal transformations are small perturbation on the basis of the generators of symmetric transformations of undisturbed system, and we have

$$
\begin{aligned}
& \xi_{0}^{\sigma}=\xi_{0}^{\sigma 0}+\rho \xi_{0}^{\sigma 1}+\rho^{2} \xi_{0}^{\sigma 2}+\cdots, \\
& \xi_{\mu}^{\sigma}=\xi_{\mu}^{\sigma 0}+\rho \xi_{\mu}^{\sigma 1}+\rho^{2} \xi_{\mu}^{\sigma 2}+\cdots .
\end{aligned}
$$

And the gauge function $G^{\sigma}$ causes a small perturbation correspondingly, and we have

$$
G^{\sigma}=G^{\sigma 0}+\rho G^{\sigma 1}+\rho^{2} G^{\sigma 2}+\cdots .
$$

Hence we have the following.

Theorem 6. For the Birkhoffian system (3), which is disturbed by the small perturbation $\rho Q_{\mu}$, if there is a gauge function $G^{\sigma j}\left(\tau, a^{\nu}\right)$ that makes the generators $\xi_{0}^{\sigma j}\left(\tau, a^{\nu}\right)$ and $\xi_{\mu}^{\sigma j}\left(\tau, a^{\nu}\right)$ of infinitesimal transformations satisfy

$$
\begin{aligned}
& \left(\frac{\partial R_{\mu}}{\partial \tau} \dot{a}^{\mu}-\frac{\partial B}{\partial \tau}\right) \xi_{0}^{\sigma j}+\left(\frac{\partial R_{\mu}}{\partial a^{\nu}} \dot{a}^{\mu}-\frac{\partial B}{\partial a^{\nu}}\right) \xi_{\nu}^{\sigma j} \\
& \quad+R_{\mu}\left(\dot{\xi}_{\mu}^{\sigma j}-\dot{a}^{\mu} \dot{\xi}_{0}^{\sigma j}\right)+\left(R_{\mu} \dot{a}^{\mu}-B\right) \\
& \quad \cdot\left(\dot{\xi}_{0}^{\sigma j}+\frac{(1-\alpha) \sinh \tau}{\cosh t-\cosh \tau} \xi_{0}^{\sigma j}\right) \\
& \quad-Q_{\mu}\left(\xi_{\mu}^{\sigma, j-1}-\dot{a}^{\mu} \xi_{0}^{\sigma, j-1}\right) \\
& =-\dot{G}^{\sigma j}(\cosh t-\cosh \tau)^{1-\alpha}, \\
& \quad(j=0,1,2, \ldots ; \sigma=1,2, \ldots, r),
\end{aligned}
$$

where one assumes that $\xi_{0}^{\sigma,-1}=\xi_{\mu}^{\sigma,-1}=0$ when $j=0$, then

$$
\begin{aligned}
& I_{z}^{\sigma} \\
& \quad=\sum_{j=0}^{z} \rho^{j}\left[\left(R_{\mu} \xi_{\mu}^{\sigma j}-B \xi_{0}^{\sigma j}\right)(\cosh t-\cosh \tau)^{\alpha-1}+G^{\sigma j}\right]
\end{aligned}
$$

is a zth-order adiabatic invariant of the system.

Proof. Taking the derivative of $I_{z}^{\sigma}$ with respect to $\tau$ and utilizing the conditions (28) and the disturbed differential equations of motion (25), we have

$$
\begin{aligned}
& \frac{d I_{z}^{\sigma}}{d \tau}=\sum_{j=0}^{z} \rho^{j}\left[\left(\frac{\partial R_{\mu}}{\partial \tau} \xi_{\mu}^{\sigma j}+\frac{\partial R_{\mu}}{\partial a^{\nu}} \dot{a}^{\nu} \xi_{\mu}^{\sigma j}+R_{\mu} \dot{\xi}_{\mu}^{\sigma j}-\frac{\partial B}{\partial \tau} \xi_{0}^{\sigma j}\right.\right. \\
& \left.-\frac{\partial B}{\partial a^{\nu}} \dot{a}^{\nu} \xi_{0}^{\sigma j}-B \dot{\xi}_{0}^{\sigma j}\right)(\cosh t-\cosh \tau)^{\alpha-1} \\
& +\frac{(1-\alpha) \sinh \tau}{\cosh t-\cosh \tau}\left(R_{\mu} \xi_{\mu}^{\sigma j}-B \xi_{0}^{\sigma j}\right)(\cosh t \\
& \left.-\cosh \tau)^{\alpha-1}+\dot{G}^{\sigma j}\right]=\sum_{j=0}^{z} \rho^{j}\left[\frac{\partial R_{\mu}}{\partial \tau} \xi_{\mu}^{\sigma j}+\frac{\partial R_{\mu}}{\partial a^{\nu}}\right. \\
& \cdot \dot{a}^{\nu} \xi_{\mu}^{\sigma j}+R_{\mu} \dot{\xi}_{\mu}^{\sigma j}-\frac{\partial B}{\partial \tau} \xi_{0}^{\sigma j}-\frac{\partial B}{\partial a^{\nu}} \dot{a}^{\nu} \xi_{0}^{\sigma j}-B \dot{\xi}_{0}^{\sigma j} \\
& +\frac{(1-\alpha) \sinh \tau}{\cosh t-\cosh \tau}\left(R_{\mu} \xi_{\mu}^{\sigma j}-B \xi_{0}^{\sigma j}\right)-\left(\frac{\partial R_{\mu}}{\partial \tau} \dot{a}^{\mu}\right. \\
& \left.-\frac{\partial B}{\partial \tau}\right) \xi_{0}^{\sigma j}-\left(\frac{\partial R_{\mu}}{\partial a^{\nu}} \dot{a}^{\mu}-\frac{\partial B}{\partial a^{\nu}}\right) \xi_{\nu}^{\sigma j}-R_{\mu}\left(\dot{\xi}_{\mu}^{\sigma j}\right. \\
& \left.-\dot{a}^{\mu} \dot{\xi}_{0}^{\sigma j}\right)-\left(R_{\mu} \dot{a}^{\mu}-B\right)\left(\dot{\xi}_{0}^{\sigma j}\right. \\
& \left.\left.+\frac{(1-\alpha) \sinh \tau}{\cosh t-\cosh \tau} \xi_{0}^{\sigma j}\right)+Q_{\mu}\left(\xi_{\mu}^{\sigma, j-1}-\dot{a}^{\mu} \xi_{0}^{\sigma, j-1}\right)\right] \\
& \cdot(\cosh t-\cosh \tau)^{\alpha-1} \\
& =\sum_{j=0}^{z} \rho^{j}\left\{\left[-\left(\frac{\partial R_{v}}{\partial a^{\mu}}-\frac{\partial R_{\mu}}{\partial a^{\nu}}\right) \dot{a}^{\nu}+\frac{\partial B}{\partial a^{\mu}}+\frac{\partial R_{\mu}}{\partial \tau}\right.\right.
\end{aligned}
$$




$$
\begin{aligned}
& \left.+\frac{(1-\alpha) \sinh \tau}{\cosh t-\cosh \tau} R_{\mu}\right]\left(\xi_{\mu}^{\sigma j}-\dot{a}^{\mu} \xi_{0}^{\sigma j}\right)+Q_{\mu}\left(\xi_{\mu}^{\sigma, j-1}\right. \\
& \left.\left.-\dot{a}^{\mu} \xi_{0}^{\sigma, j-1}\right)\right\}(\cosh t-\cosh \tau)^{\alpha-1} \\
& =\sum_{j=0}^{z} \rho^{j}\left[-\rho Q_{\mu}\left(\xi_{\mu}^{\sigma j}-\dot{a}^{\mu} \xi_{0}^{\sigma j}\right)+Q_{\mu}\left(\xi_{\mu}^{\sigma, j-1}\right.\right. \\
& \left.\left.-\dot{a}^{\mu} \xi_{0}^{\sigma, j-1}\right)\right](\cosh t-\cosh \tau)^{\alpha-1}=-\rho^{z+1} Q_{\mu}\left(\xi_{\mu}^{\sigma z}\right. \\
& \left.-\dot{a}^{\mu} \xi_{0}^{\sigma z}\right)(\cosh t-\cosh \tau)^{\alpha-1} .
\end{aligned}
$$

According to the definition [29, 30] of high-order adiabatic invariant, $I_{z}^{\sigma}$ is a $z$ th-order adiabatic invariant of the Birkhoffian system under the El-Nabulsi dynamics model. This completes the proof.

According to (30), if $Q_{\mu}=0$, then $d I_{z}^{\sigma} / d \tau=0$. Therefore, the exact invariant of the Birkhoffian system is a special adiabatic invariant.

Now we give several special cases of Theorem 6. Firstly, if we take $\alpha=1$, then Theorem 6 gives high-order adiabatic invariants for classical Birkhoffian system, and we have the following theorem.

Theorem 7. For the classical Birkhoffian system (4), which is disturbed by the small perturbation $\rho Q_{\mu}$, if there is a gauge function $G^{\sigma j}\left(\tau, a^{\nu}\right)$ that makes the generators $\xi_{0}^{\sigma j}\left(\tau, a^{\nu}\right)$ and $\xi_{\mu}^{\sigma j}\left(\tau, a^{\nu}\right)$ of infinitesimal transformations satisfy

$$
\begin{aligned}
& \left(\frac{\partial R_{\mu}}{\partial \tau} \dot{a}^{\mu}-\frac{\partial B}{\partial \tau}\right) \xi_{0}^{\sigma j}+\left(\frac{\partial R_{\mu}}{\partial a^{\nu}} \dot{a}^{\mu}-\frac{\partial B}{\partial a^{\nu}}\right) \xi_{v}^{\sigma j} \\
& \quad+R_{\mu}\left(\dot{\xi}_{\mu}^{\sigma j}-\dot{a}^{\mu} \dot{\xi}_{0}^{\sigma j}\right)+\left(R_{\mu} \dot{a}^{\mu}-B\right) \dot{\xi}_{0}^{\sigma j} \\
& \quad-Q_{\mu}\left(\xi_{\mu}^{\sigma, j-1}-\dot{a}^{\mu} \xi_{0}^{\sigma, j-1}\right) \\
& =-\dot{G}^{\sigma j}(\cosh t-\cosh \tau)^{1-\alpha}, \\
& \quad(j=0,1,2, \ldots ; \sigma=1,2, \ldots, r),
\end{aligned}
$$

where one assumes that $\xi_{0}^{\sigma,-1}=\xi_{\mu}^{\sigma,-1}=0$ when $j=0$, then

$$
I_{z}^{\sigma}=\sum_{j=0}^{z} \rho^{j}\left(R_{\mu} \xi_{\mu}^{\sigma j}-B \xi_{0}^{\sigma j}+G^{\sigma j}\right)
$$

is a zth-order adiabatic invariants of the system.

Secondly, let us discuss a nonconservative Hamiltonian system under the El-Nabulsi dynamics model. Theorem 6 is reduced to the following theorem.

Theorem 8. For the nonconservative Hamiltonian system (6), which is disturbed by the small perturbation $\rho Q_{k}$, if there is a gauge function $G^{\sigma j}\left(\tau, q^{s}, p_{s}\right)$ that makes the generators $\xi_{0}^{\sigma j}\left(\tau, q^{s}, p_{s}\right)$ and $\xi_{k}^{\sigma j}\left(\tau, q^{s}, p_{s}\right)$ of infinitesimal transformations satisfy

$$
\begin{aligned}
& -\frac{\partial H}{\partial \tau} \xi_{0}^{\sigma j}-\frac{\partial H}{\partial q^{k}} \xi_{k}^{\sigma j}+\left(\dot{q}^{k}-\frac{\partial H}{\partial p_{k}}\right) \eta_{k}^{\sigma j} \\
& \quad+p_{k}\left(\dot{\xi}_{k}^{\sigma j}-\dot{q}^{k} \dot{\xi}_{0}^{\sigma j}\right)+\left(p_{k} \dot{q}^{k}-H\right) \\
& \quad \cdot\left(\dot{\xi}_{0}^{\sigma j}+\frac{(1-\alpha) \sinh \tau}{\cosh t-\cosh \tau} \xi_{0}^{\sigma j}\right) \\
& \quad+Q_{k}\left(\xi_{k}^{\sigma, j-1}-\dot{q}^{k} \xi_{0}^{\sigma, j-1}\right) \\
& =-\dot{G}^{\sigma j}(\cosh t-\cosh \tau)^{1-\alpha}, \\
& \quad(j=0,1,2, \ldots ; \sigma=1,2, \ldots, r)
\end{aligned}
$$

where one assumes that $\xi_{0}^{\sigma,-1}=\xi_{k}^{\sigma,-1}=0$ when $j=0$, then

$$
\begin{aligned}
& I_{z}^{\sigma} \\
& \quad=\sum_{j=0}^{z} \rho^{j}\left[\left(p_{k} \xi_{k}^{\sigma j}-H \xi_{0}^{\sigma j}\right)(\cosh t-\cosh \tau)^{\alpha-1}+G^{\sigma j}\right]
\end{aligned}
$$

is a zth-order adiabatic invariants of the system.

Again, let us discuss a nonconservative Lagrange system under the El-Nabulsi dynamics model. Theorem 6 is reduced to the following theorem.

Theorem 9. For the nonconservative Lagrange system (8), which is disturbed by the small perturbation $\rho Q_{k}$, if there is a gauge function $G^{\sigma j}\left(\tau, q^{s}, \dot{q}^{s}\right)$ that makes the generators $\xi_{0}^{\sigma j}\left(\tau, q^{s}, \dot{q}^{s}\right)$ and $\xi_{k}^{\sigma j}\left(\tau, q^{s}, \dot{q}^{s}\right)$ of infinitesimal transformations satisfy

$$
\begin{aligned}
& \frac{\partial L}{\partial \tau} \xi_{0}^{\sigma j}+\frac{\partial L}{\partial q^{k}} \xi_{k}^{\sigma j}+\frac{\partial L}{\partial \dot{q}^{k}}\left(\dot{\xi}_{k}^{\sigma j}-\dot{q}^{k} \dot{\xi}_{0}^{\sigma j}\right) \\
& +L\left(\dot{\xi}_{0}^{\sigma j}+\frac{(1-\alpha) \sinh \tau}{\cosh t-\cosh \tau} \xi_{0}^{\sigma j}\right) \\
& +Q_{k}\left(\xi_{k}^{\sigma, j-1}-\dot{q}^{k} \xi_{0}^{\sigma, j-1}\right) \\
& =-\dot{G}^{\sigma j}(\cosh t-\cosh \tau)^{1-\alpha}, \\
& \quad(j=0,1,2, \ldots ; \sigma=1,2, \ldots, r),
\end{aligned}
$$

where one assumes that $\xi_{0}^{\sigma,-1}=\xi_{k}^{\sigma,-1}=0$ when $j=0$, then

$$
\begin{gathered}
I_{z}^{\sigma}=\sum_{j=0}^{z} \rho^{j}\left\{\left[L \xi_{0}^{\sigma j}+\frac{\partial L}{\partial \dot{q}^{k}}\left(\xi_{k}^{\sigma j}-\dot{q}^{k} \xi_{0}^{\sigma j}\right)\right]\right. \\
\left.\cdot(\cosh t-\cosh \tau)^{\alpha-1}+G^{\sigma j}\right\}
\end{gathered}
$$

is a zth-order adiabatic invariants of the system. 


\section{An Illustrative Example}

As an example, let us consider the problem of HojmanUrrutia [2]. The Birkhoffian $B$ and Birkhoff's functions $R_{\mu}$ are $[2,3]$

$$
\begin{aligned}
B & =\frac{1}{2}\left[\left(a^{3}\right)^{2}+2 a^{2} a^{3}-\left(a^{4}\right)^{2}\right], \\
R_{1} & =a^{2}+a^{3}, \\
R_{2} & =0 \\
R_{3} & =a^{4}, \\
R_{4} & =0 .
\end{aligned}
$$

We try to study the perturbation to Noether symmetries and the adiabatic invariants of the system under El-Nabulsi dynamics model from extended exponentially fractional integral.

According to the results given by Santilli [2], system (37) admits the Birkhoffian representation. But this system is not essentially self-adjoint, so it does not admit a Hamiltonian representation. The differential equations (3) of motion of the system give

$$
\begin{aligned}
-\dot{a}^{2}-\dot{a}^{3} & =\frac{(1-\alpha) \sinh \tau}{\cosh t-\cosh \tau}\left(a^{2}+a^{3}\right), \\
\dot{a}^{1}-a^{3} & =0, \\
\dot{a}^{1}-\dot{a}^{4}-a^{2}-a^{3} & =\frac{(1-\alpha) \sinh \tau}{\cosh t-\cosh \tau} a^{4}, \\
\dot{a}^{3}+a^{4} & =0 .
\end{aligned}
$$

Condition (16) gives

$$
\begin{aligned}
& \left(\dot{a}^{1}-a^{3}\right) \xi_{2}^{\sigma}+\left(\dot{a}^{1}-a^{2}-a^{3}\right) \xi_{3}^{\sigma}+\left(\dot{a}^{3}+a^{4}\right) \xi_{4}^{\sigma} \\
& +\left(a^{2}+a^{3}\right)\left(\dot{\xi}_{1}^{\sigma}-\dot{a}^{1} \dot{\xi}_{0}^{\sigma}\right)+a^{4}\left(\dot{\xi}_{3}^{\sigma}-\dot{a}^{3} \dot{\xi}_{0}^{\sigma}\right) \\
& +\left(a^{2} \dot{a}^{1}+a^{3} \dot{a}^{1}+a^{4} \dot{a}^{3}-B\right) \\
& \quad \cdot\left(\dot{\xi}_{0}^{\sigma}+\frac{(1-\alpha) \sinh \tau}{\cosh t-\cosh \tau} \xi_{0}^{\sigma}\right) \\
& =-\dot{G}^{\sigma}(\cosh t-\cosh \tau)^{1-\alpha} .
\end{aligned}
$$

By use of (38), (39) has the following solutions:

$$
\begin{aligned}
& \xi_{0}^{10}=0, \\
& \xi_{1}^{10}=1, \\
& \xi_{2}^{10}=0, \\
& \xi_{3}^{10}=0, \\
& \xi_{4}^{10}=0, \\
& G^{10}=0,
\end{aligned}
$$

$$
\begin{aligned}
\xi_{0}^{20} & =(\cosh t-\cosh \tau)^{1-\alpha} \\
\xi_{1}^{20} & =2, \\
\xi_{2}^{20} & =0 \\
\xi_{3}^{20} & =0 \\
\xi_{4}^{20} & =0 \\
G^{20} & =\frac{1}{2}\left(a^{3}\right)^{2}+a^{2} a^{3}-\frac{1}{2}\left(a^{4}\right)^{2}, \\
\xi_{0}^{30} & =\left(\cosh t-\cosh ^{1-\alpha}\right)^{1-\alpha} \\
\xi_{1}^{30} & =0 \\
\xi_{2}^{30} & =0 \\
\xi_{3}^{30} & =0 \\
\xi_{4}^{30} & =0 \\
G^{30} & =\frac{1}{2}\left(a^{3}\right)^{2}+a^{2} a^{3}-\frac{1}{2}\left(a^{4}\right)^{2} .
\end{aligned}
$$

Obviously, generators (40) correspond to a Noether symmetric transformation of the system under El-Nabulsi dynamics model, and generators (41) and (42) correspond to Noether quasisymmetric transformations. According to Theorem 2, in correspondence with generators (40), (41), and (42), formula (17) gives, respectively,

$$
\begin{aligned}
& I_{0}^{1}=\left(a^{2}+a^{3}\right)(\cosh t-\cosh \tau)^{\alpha-1}=c^{1}, \\
& I_{0}^{2}=2\left(a^{2}+a^{3}\right)(\cosh t-\cosh \tau)^{\alpha-1}=c^{2}, \\
& I_{0}^{3}=0 .
\end{aligned}
$$

Conserved quantities (43)-(45) are the exact invariants. Formula (45) shows that the infinitesimal transformation corresponding to generators (42) is trivial. And when $\alpha=1$, the conserved quantities above are reduced to the Noether conserved quantities for classical Birkhoffian system.

Now let us study the adiabatic invariants of the system. Suppose that the system is disturbed by the following small forces of perturbation:

$$
\begin{aligned}
& \rho Q_{1}=\rho a^{2}, \\
& \rho Q_{2}=\rho a^{1}, \\
& \rho Q_{3}=\rho a^{3}, \\
& \rho Q_{4}=\rho a^{4} .
\end{aligned}
$$

Under the action of (46), the disturbed differential equations of motion (25) give

$$
\begin{aligned}
-\dot{a}^{2}-\dot{a}^{3} & =\frac{(1-\alpha) \sinh \tau}{\cosh t-\cosh \tau}\left(a^{2}+a^{3}\right)+\rho a^{2}, \\
\dot{a}^{1}-a^{3} & =\rho a^{1},
\end{aligned}
$$




$$
\begin{aligned}
\dot{a}^{1}-\dot{a}^{4}-a^{2}-a^{3} & =\frac{(1-\alpha) \sinh \tau}{\cosh t-\cosh \tau} a^{4}+\rho a^{3}, \\
\dot{a}^{3}+a^{4} & =\rho a^{4} .
\end{aligned}
$$

Condition (28) gives

$$
\begin{aligned}
& \left(\dot{a}^{1}-a^{3}\right) \xi_{2}^{\sigma 1}+\left(\dot{a}^{1}-a^{3}-a^{2}\right) \xi_{3}^{\sigma 1}+\left(\dot{a}^{3}+a^{4}\right) \xi_{4}^{\sigma 1} \\
& +\left(a^{2}+a^{3}\right)\left(\dot{\xi}_{1}^{\sigma 1}-\dot{a}^{1} \dot{\xi}_{0}^{\sigma 1}\right)+a^{4}\left(\dot{\xi}_{3}^{\sigma 1}-\dot{a}^{3} \dot{\xi}_{0}^{\sigma 1}\right) \\
& +\left(a^{2} \dot{a}^{1}+a^{3} \dot{a}^{1}+a^{4} \dot{a}^{3}-B\right) \\
& +\left(\dot{\xi}_{0}^{\sigma 1}+\frac{(1-\alpha) \sinh \tau}{\cosh t-\cosh \tau} \xi_{0}^{\sigma 1}\right)-a^{2}\left(\xi_{1}^{\sigma 0}-\dot{a}^{1} \xi_{0}^{\sigma 0}\right) \\
& -a^{1}\left(\xi_{2}^{\sigma 0}-\dot{a}^{2} \xi_{0}^{\sigma 0}\right)-a^{3}\left(\xi_{3}^{\sigma 0}-\dot{a}^{3} \xi_{0}^{\sigma 0}\right) \\
& -a^{4}\left(\xi_{4}^{\sigma 0}-\dot{a}^{4} \xi_{0}^{\sigma 0}\right)=-\dot{G}^{\sigma 1}(\cosh t-\cosh \tau)^{1-\alpha}
\end{aligned}
$$

By using generators (40), (48) has a solution

$$
\begin{aligned}
& \xi_{0}^{11}=0, \\
& \xi_{1}^{11}=0, \\
& \xi_{2}^{11}=1, \\
& \xi_{3}^{11}=-1, \\
& \xi_{4}^{11}=0, \\
& G^{11}=0 .
\end{aligned}
$$

According to Theorem 6, in correspondence with generators (49) and (40), the system has a first-order adiabatic invariant as follows:

$$
\begin{aligned}
I_{1}^{1}= & \left(a^{2}+a^{3}\right)(\cosh t-\cosh \tau)^{\alpha-1} \\
& -\rho a^{4}(\cosh t-\cosh \tau)^{\alpha-1}
\end{aligned}
$$

By using generators (41), (48) has a solution

$$
\begin{aligned}
& \xi_{0}^{21}=0, \\
& \xi_{1}^{21}=0, \\
& \xi_{2}^{21}=2, \\
& \xi_{3}^{21}=-2, \\
& \xi_{4}^{21}=0, \\
& G^{21}=-\frac{1}{2}\left(a^{3}\right)^{2}-a^{1} a^{2}-\frac{1}{2}\left(a^{4}\right)^{2} .
\end{aligned}
$$

According to Theorem 6, in correspondence with generators (51) and (41), the system has a first-order adiabatic invariant as follows:

$$
\begin{aligned}
I_{1}^{2} & =2\left(a^{2}+a^{3}\right)(\cosh t-\cosh \tau)^{\alpha-1} \\
& -\rho\left[2 a^{4}(\cosh t-\cosh \tau)^{\alpha-1}+\frac{1}{2}\left(a^{3}\right)^{2}+a^{1} a^{2}\right. \\
& \left.+\frac{1}{2}\left(a^{4}\right)^{2}\right] .
\end{aligned}
$$

By using generators (42), (48) has a solution

$$
\begin{aligned}
& \xi_{0}^{31}=0, \\
& \xi_{1}^{31}=1, \\
& \xi_{2}^{31}=0, \\
& \xi_{3}^{31}=0, \\
& \xi_{4}^{31}=0, \\
& G^{31}=-\frac{1}{2}\left(a^{3}\right)^{2}-a^{1} a^{2}-\frac{1}{2}\left(a^{4}\right)^{2} .
\end{aligned}
$$

According to Theorem 6, in correspondence with generators (54) and (42), the system has a first-order adiabatic invariant as follows:

$$
\begin{aligned}
I_{1}^{3} & =\rho\left[\left(a^{2}+a^{3}\right)(\cosh t-\cosh \tau)^{\alpha-1}-\frac{1}{2}\left(a^{3}\right)^{2}\right. \\
& \left.-a^{1} a^{2}-\frac{1}{2}\left(a^{4}\right)^{2}\right] .
\end{aligned}
$$

Furthermore, we can find higher-order adiabatic invariants of the system.

\section{Conclusions}

Symmetries are important and common properties of a dynamical system, and the perturbation to symmetries and corresponding invariants under the action of small forces of perturbation have an intimate relationship with the integrability of the system. An adiabatic invariant comes from the essential characteristics of the system, and it is more than just products of a Hamiltonian system. In this paper, we extended the El-Nabulsi dynamics model which is based on extended exponentially fractional integral to a Birkhoffian system, established the Noether theory of the Birkhoffian system under the El-Nabulsi model, and studied the problem of the perturbation to Noether symmetries and the adiabatic invariants for the disturbed Birkhoffian system. The methods and results of this paper are of universal significance. The corresponding consequences of classical Birkhoffian system are special cases of this paper, as well as the nonconservative Hamiltonian system and the nonconservative Lagrangian system under El-Nabulsi dynamics models. Further work could include the extension and the application of El-Nabulsi dynamics models to various types of constrained mechanical 
systems and the study of geometrical aspects and symplectic manifolds as well as some application to quantum mechanics and field theory.

\section{Conflict of Interests}

The author declares that there is no conflict of interests regarding the publication of this paper.

\section{Acknowledgments}

This work is supported by the National Natural Science Foundation of China (Grants nos. 10972151 and 11272227).

\section{References}

[1] G. D. Birkhoff, Dynamical Systems, AMS College Publication, Providence, RI, USA, 1927.

[2] R. M. Santilli, Foundations of Theoretical Mechanics II, Springer, New York, NY, USA, 1983.

[3] F. X. Mei, R. C. Shi, Y. F. Zhang, and H. B. Wu, Dynamics of Birkhoffian System, Beijing Institute of Technology Press, Beijing, China, 1996, (Chinese).

[4] A. S. Galiullan, Analytical Dynamics, Nauka, Moscow, Russia, 1989, (Russian).

[5] A. S. Galiullin, G. G. Gafarov, R. P. Malaishka, and A. M. Khwan, Analytical Dynamics of Helmholtz, Birkhoff and Nambu Systems, UFN, Moscow, Russia, 1997, (Russian).

[6] F. X. Mei, "The progress of research on dynamics of Birkhoff's system," Advances in Mechanics, vol. 27, no. 4, pp. 436-446, 1997 (Chinese).

[7] F.-X. Mei, “On the Birkhoffian mechanics," International Journal of Non-Linear Mechanics, vol. 36, no. 5, pp. 817-834, 2001.

[8] F. X. Mei, Dynamics of Generalized Birkhoffian System, Science Press, Beijing, China, 2013, (Chinese).

[9] F. Riewe, "Nonconservative Lagrangian and Hamiltonian mechanics," Physical Review E, vol. 53, no. 2, pp. 1890-1899, 1996.

[10] F. Riewe, "Mechanics with fractional derivatives," Physical Review E. Statistical, Nonlinear, and Soft Matter Physics, vol. 55, no. 3, pp. 3581-3592, 1997.

[11] M. Klimek, "Fractional sequential mechanics-models with symmetric fractional derivative," Czechoslovak Journal of Physics, vol. 51, no. 12, pp. 1348-1354, 2001.

[12] O. P. Agrawal, "Formulation of Euler-Lagrange equations for fractional variational problems," Journal of Mathematical Analysis and Applications, vol. 272, no. 1, pp. 368-379, 2002.

[13] O. P. Agrawal, "Fractional variational calculus in terms of Riesz fractional derivatives," Journal of Physics A: Mathematical and Theoretical, vol. 40, no. 24, pp. 6287-6303, 2007.

[14] T. M. Atanacković, S. Konjik, and S. Pilipović, "Variational problems with fractional derivatives: Euler-Lagrange equations," Journal of Physics A: Mathematical and Theoretical, vol. 41, no. 9, Article ID 095201, 12 pages, 2008.

[15] T. M. Atanacković, S. Konjik, S. Pilipović, and S. Simić, "Variational problems with fractional derivatives: invariance conditions and Nöther's theorem," Nonlinear Analysis: Theory, Methods \& Applications, vol. 71, no. 5-6, pp. 1504-1517, 2009.
[16] D. Baleanu and T. Avkar, "Lagrangians with linear velocities within Riemann-Liouville fractional derivatives," Nuovo Cimento B, vol. 119, no. 1, pp. 73-79, 2004.

[17] D. Baleanu, T. Maaraba, and F. Jarad, "Fractional variational principles with delay," Journal of Physics A: Mathematical and Theoretical, vol. 41, no. 31, Article ID 315403, 8 pages, 2008.

[18] R. Almeida and D. F. M. Torres, "Necessary and sufficient conditions for the fractional calculus of variations with Caputo derivatives," Communications in Nonlinear Science and Numerical Simulation, vol. 16, no. 3, pp. 1490-1500, 2011.

[19] A. B. Malinowska and D. F. Torres, Introduction to the Fractional Calculus of Variations, Imperial College Press, London, UK, 2012.

[20] J. Cresson, "Fractional embedding of differential operators and Lagrangian systems," Journal of Mathematical Physics, vol. 48, no. 3, Article ID 033504, 34 pages, 2007.

[21] R. A. El-Nabulsi, "A fractional approach to nonconservative Lagrangian dynamical systems," Fizika A, vol. 14, no. 4, pp. 289298, 2005.

[22] R. A. El-Nabulsi, "A fractional action-like variational approach of some classical, quantum and geometrical dynamics," International Journal of Applied Mathematics, vol. 17, no. 3, pp. 299-317, 2005.

[23] R. A. El-Nabulsi, "Fractional variational problems from extended exponentially fractional integral," Applied Mathematics and Computation, vol. 217, no. 22, pp. 9492-9496, 2011.

[24] R. A. El-Nabulsi, "A periodic functional approach to the calculus of variations and the problem of time-dependent damped harmonic oscillators," Applied Mathematics Letters, vol. 24, no. 10, pp. 1647-1653, 2011.

[25] G. S. F. Frederico and D. F. M. Torres, "Constants of motion for fractional action-like variational problems," International Journal of Applied Mathematics, vol. 19, no. 1, pp. 97-104, 2006.

[26] G. S. F. Frederico and D. F. M. Torres, "Non-conservative Noether's theorem for fractional action-like variational problems with intrinsic and observer times," International Journal of Ecological Economics \& Statistics, vol. 9, no. 7, pp. 74-82, 2007.

[27] Y. Zhang and Y. Zhou, "Symmetries and conserved quantities for fractional action-like Pfaffian variational problems," Nonlinear Dynamics, vol. 73, no. 1-2, pp. 783-793, 2013.

[28] J. F. Ding and Y. Zhang, "Noether symmetries for the ElNabulsi-Pfaff variational problem from extended exponentially fractional integral," Acta Scientiarum Naturalium Universitatis Sunyatseni, vol. 53, no. 6, pp. 150-154, 2014 (Chinese).

[29] V. I. Arnold, V. V. Kozlov, and A. I. Neishtadt, Mathematical Aspects of Classical and Celestial Mechanics, Encyclopaedia of Mathematical Sciences, Springer, Berlin, Germany, 3rd edition, 2006.

[30] F. X. Mei, D. Liu, and Y. Luo, Advanced Analytical Mechanics, Beijing Institute of Technology Press, Beijing, China, 1991, (Chinese).

[31] Y. Y. Zhao and F. X. Mei, "Exact invariant and adiabatic invariant of a general dynamical system," Acta Mechanica Sinica, vol. 28, no. 2, pp. 207-216, 1996 (Chinese).

[32] X.-W. Chen, Y.-M. Li, and Y.-H. Zhao, "Lie symmetries, perturbation to symmetries and adiabatic invariants of Lagrange system," Physics Letters A, vol. 337, no. 4-6, pp. 274-278, 2005.

[33] Y. Zhang, "A new type of adiabatic invariants for Birkhoffian system," Acta Physica Sinica, vol. 55, no. 8, pp. 3833-3837, 2006.

[34] Z. J. Li, W. A. Jiang, and S. K. Luo, "Lie symmetries, symmetrical perturbation and a new adiabatic invariant for disturbed 
nonholonomic systems," Nonlinear Dynamics, vol. 67, no. 1, pp. 445-455, 2012.

[35] J. Chen and Y. Zhang, "Perturbation to Noether symmetries and adiabatic invariants for disturbed Hamiltonian systems based on El-NABulsi nonconservative dynamics model," Nonlinear Dynamics, vol. 77, no. 1-2, pp. 353-360, 2014.

[36] J. Chen and Y. Zhang, "Perturbation to Noether symmetries and adiabatic invariants for Birkhoffian systems based on El-Nabulsi dynamical models," Acta Physica Sinica, vol. 63, no. 10, Article ID 104501, 7 pages, 2014.

[37] R. A. El-Nabulsi, "Fractional quantum euler-cauchy equation in the schrödinger picture, complexified harmonic oscillators and emergence of complexified lagrangian and hamiltonian dynamics," Modern Physics Letters B, vol. 23, no. 28, pp. 33693386, 2009.

[38] R. A. El-Nabulsi, "The fractional calculus of variations from extended Erdélyi-Kober operator," International Journal of Modern Physics B, vol. 23, no. 16, pp. 3349-3361, 2009. 


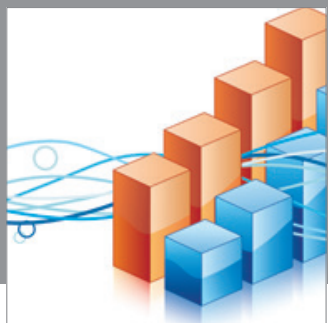

Advances in

Operations Research

mansans

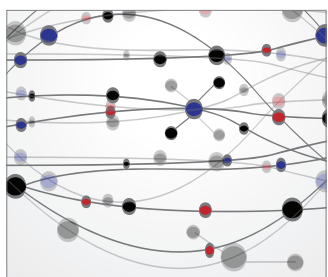

The Scientific World Journal
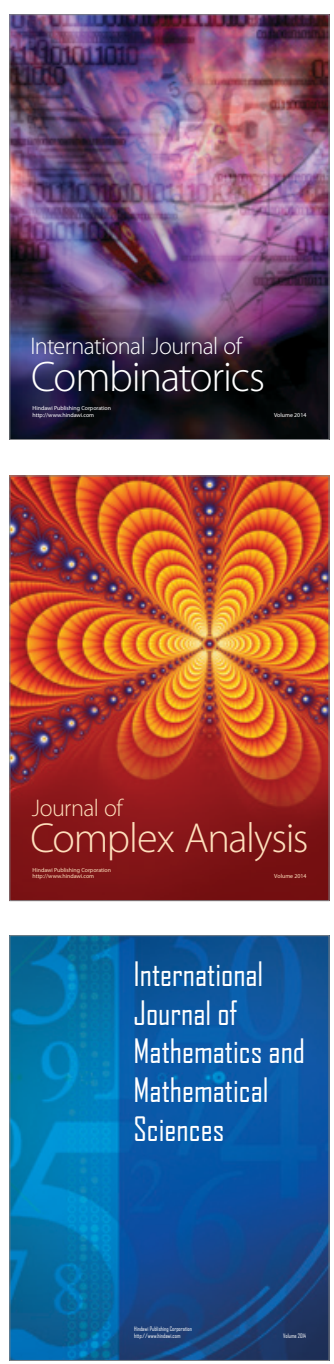
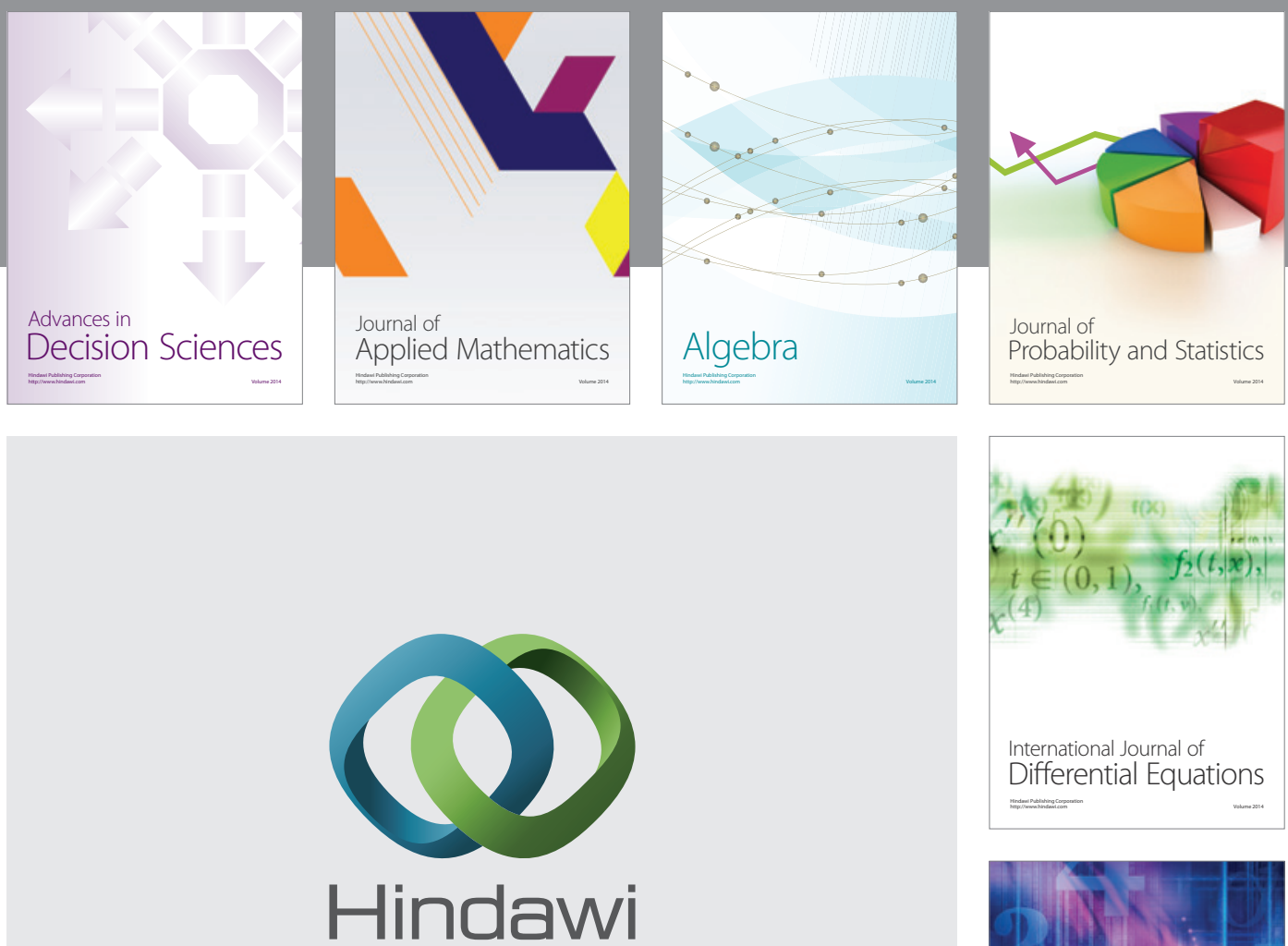

Submit your manuscripts at http://www.hindawi.com
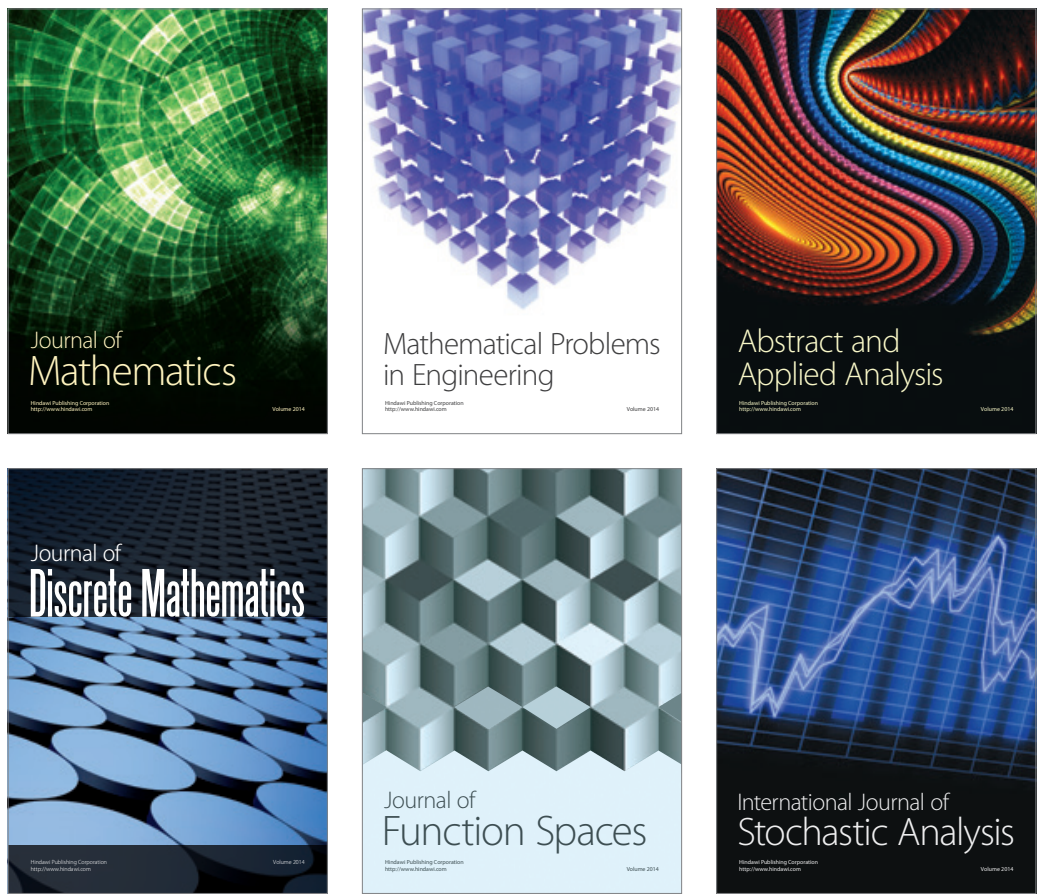

Journal of

Function Spaces

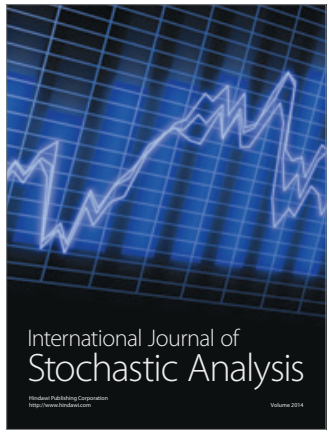

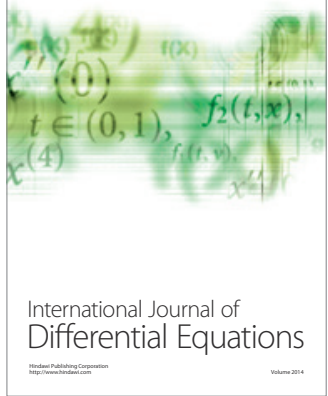
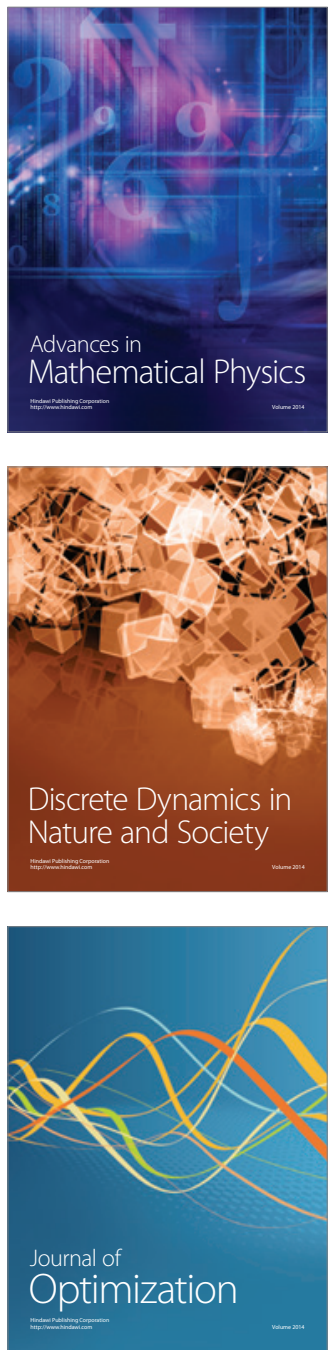